\title{
Parametric Resonance in Electrostatically Actuated Micromirrors
}

\author{
Attilio Frangi, Andrea Guerrieri, Roberto Carminati, and Gianluca Mendicino
}

\begin{abstract}
We consider an electrostatically actuated torsional micromirror, a key element of recent optical microdevices. The mechanical response is analyzed with specific emphasis on its nonlinear features. We show that the mirror motion is an example of parametric resonance, activated when the drive frequency is twice the natural frequency of the system. The numerical model, solved with a continuation approach, is validated with very good accuracy through an extensive experimental campaign.
\end{abstract}

Index Terms-Continuation approaches, Mathieu equation, micromirrors, microoptoelectromechanical systems (MOEMS), parametric resonance.

\section{INTRODUCTION}

M ICROMIRRORS are emerging as a very successful component (see, e.g., [1]) within the family of microoptoelectromechanical systems (MOEMS). MOEMS, in general, are microelectromechanical systems (MEMS) merged with microoptics and involve sensing or manipulating optical signals on a very small size scale, using integrated mechanical, optical, and electrical systems. Coupling all these technologies is paving the way to several devices such as, e.g., optical switches, digital micromirrors and dynamic displays, bistable mirrors, laser microscanners, optical shutters, microspectrometers, and microlenses.

Among these, the pico-projector is a response to the emergent need of compact portable devices to project digital images onto any nearby external viewing surfaces. This optical engine is composed of three lasers diodes (i.e., red, green, and blue colors), two micromirrors for the laser beams deflection, and some passive optical elements like lenses and microfilters. Another example is provided by the microscanner for real-time 3 -D shape measurement of moving objects. Known devices are formed using three components: a conventional color CMOS image sensor camera, an infrared images sensor, an infrared light projector plus an image processor. The infrared light projector emits a structured pattern of infrared light, which is re-

Manuscript received April 21, 2016; revised July 26, 2016; accepted September 11, 2016. Date of publication October 5, 2016; date of current version January 10,2017 . This work was developed in the framework of Lab4MEMSII, a project launched by the ENIAC Joint Undertaking, Project ID: 621176-2 (ENIAC Call 2013-2).

A. Frangi and A. Guerrieri are with the Department of Civil and Environmental Engineering, Politecnico di Milano, 20133 Milan Italy (e-mail: attilio.frangi@ polimi.it; andrea.guerrieri@polimi.it).

R. Carminati and G. Mendicino are with the MSH Division, STMicroelectronics, 20010 Cornaredo Italy (e-mail: roberto.carminati@st.com; gianluca.mendicino@st.com).

Color versions of one or more of the figures in this paper are available online at http://ieeexplore.ieee.org

Digital Object Identifier 10.1109/TIE.2016.2615274 flected back from the scene and is detected by the infrared image sensor, making it possible to reconstruct a 3-D scene. The light projector contains an IR laser diode, a line lens, and a MEMS resonant micromirror device all mounted onto a small optical bench.

The resonant micromirror is hence an important element for both applications. A typical example, on which we focus in this investigation, is depicted in Fig. 1. The device features an electrostatically actuated torsional mirror, similar to that described in a recent publication [2] on the effects of fluid damping by ambient air on the large oscillations of the mirror. In this paper, we discuss and simulate the mechanical response of the torsional mirror responding in its main torsional mode and finally validate the results with an experimental campaign. We show that it is based on parametric resonance, which is a known phenomenon in the nonlinear dynamics of structures. MEMS and MOEMS provide several pieces of evidence of nonlinear phenomena, mainly induced by nonlinear terms in the electrostatic or elastic stiffnesses (see, e.g., the extensive literature cited in [3] and the specific examples for micromirrors in [4]-[6]). However, in most cases, the devices are still governed by the classical harmonic resonance. Parametric resonance has been well understood in many areas of science [3], [7]-[9], including the stability of ships, the forced motion of a swing, and Faraday surface wave patterns on water. Theory predicts that parametric resonances occur near drive frequencies of $2 \omega_{0} / n$, where $\omega_{0}$ is the system's natural frequency and $n$ is any positive integer. However, in macroscopic systems, only the first resonance region near $2 \omega_{0}$ can typically be observed, because of damping and exponential narrowing of the resonance regions with increasing $n$. An exception is described in [10], where the torsional oscillations in a single-crystal silicon MEMS have been analyzed. Five instability regions have been measured, due to the low damping, stability, and precise frequency control achievable in that system.

Microsystems provide several examples of parametric resonance, starting from the pioneering work by Rugar and Grutter [11]. Applications include mass sensing, parametric amplification (see, e.g., also [12]-[21]), and others. In particular, Rhoads et al. [16] focus on the design of a highly sensitive mass sensor and use a nonlinear generalized Mathieu equation to model the problem. Analytical results show that nonlinearity significantly changes the stability characteristics of parametric resonance. It is shown that the forms of the nonlinear terms in the damping coefficient, elastic, and electrostatic stiffness have a strong effect on the actual response of the modeled device. The system can exhibit not only the typical hardening and softening nonlinearities, but also mixed nonlinearities, wherein the response branches in the system's frequency response bend toward or away from one another near resonance. In the application of interest herein, the elastic stiffness is linear and all the nonlinearities are due to the electrostatic forcing. However, these cannot be approxi- 


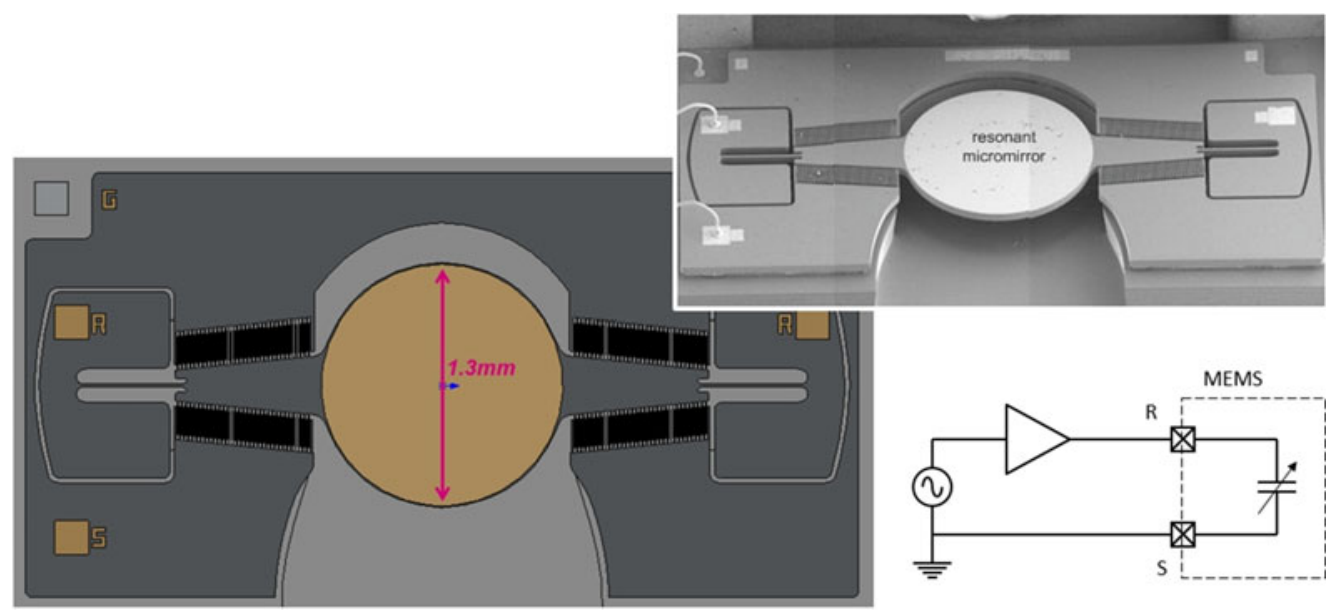

Fig. 1. Resonant micromirror: SEM image of the device, layout, and schema of the connections. R stands for rotor (rotating mirror) and S for stator which is connected to ground.

mated with linear and cubic terms due to the very large opening angles, and a very accurate numerical procedure is mandatory.

The paper is organized as follows. After introducing the mirror geometry and elaborating the dynamical model in Section II, the analytical and numerical solution of the governing equation is discussed in Section III-A. We apply continuation techniques that permit to simulate all the branches of the response, both stable and unstable. Continuation codes like AUTO [24] are the state of the art for these kind of analyses. However, periodic nonautonomous systems cannot be handled simply. While for sinusoidal voltages, this difficulty can be easily overcome, for the square wave with finite ramp speed, the only possibility is to represent the square wave as a finite sum of sinusoidal components [according to its fast Fourier transform (FFT)] inducing spurious Gibbs oscillations. We have avoided this by developing an ad-hoc code, which has been benchmarked with AUTO for the sinusoidal voltage. An extensive experimental campaign, described in Sections IV and V, validates the modeling phase for two different types of excitations: pure sinusoid and square wave, as in real operation.

\section{MicromirRor DESCRIPTION AND MOdELING}

The 2-D layout and a scanning electron microscopic (SEM) image of the micromirror are depicted in Fig. 1. The device has been fabricated with a dedicated silicon-on-insulator technology. The out-of-plane uniform thickness of the monocrystalline layer is $H=65 \mu \mathrm{m}$. The central circular reflecting surface is attached to the substrate via two coaxial beams acting as torsional springs. Metallization material for the mirror is chosen to maximize reflection in requested working optical wavelength range (typically Au for IR and Aluminum for visible light). Four sets of 33 fingers each are anchored to the trapezoidal regions directly attached to the mirror. These plates, interdigited with their stator counterparts, form a comb drive structure providing the electrostatic actuation mechanism. Sensing of the opening angle is performed, during operation, via the same comb drive electrodes. It is worth stressing that, due to symmetry, the electrostatic torque around the torsional axis vanishes for $\psi=0$, i.e., in the rest configuration, $\forall V(t)$. As will be explained next, this trivial solution becomes, however, unstable for some combinations of the input voltage and frequency and triggers the mirror rotation.

Introducing the reasonable assumption that only the torsional springs can deform elastically, while the other elements behave as rigid bodies, the following 1-D model governs the mirror response in the torsional mode:

$$
I \ddot{\psi}+B \dot{\psi}+K \psi=\frac{\varepsilon_{0}}{2} \frac{\partial C}{\partial \psi} V^{2}(t)
$$

where $I=2.375 \times 10^{10} \mathrm{ng} \cdot \mu \mathrm{m}^{2}$ is the inertia around the torsional axis; $K=2.704 \times 10^{7} \mu \mathrm{N} \cdot \mu \mathrm{m}$ is the torsional stiffness of the springs; $B=1.448 \times 10^{6} \mu \mathrm{N} \cdot \mu \mathrm{m} \cdot \mu \mathrm{s}$ is a damping coefficient, and $C=\varepsilon_{0} C_{g}$ is the system capacity, with $\varepsilon_{0}=8.85 \times 10^{-6} \mathrm{pF} / \mu \mathrm{m}$, and $C_{g}$, measured in micrometers, is a purely geometrical feature that requires numerical simulations and is commented next. The voltage $V$ is given in volts, and the time in (1) is measured in microseconds. All the values are consistent with the MEMS-like system: $\mu \mathrm{s}$ for time, ng $\left(10^{-9} \mathrm{~g}\right)$ for mass, $\mu \mathrm{m}$ for length, $\mu \mathrm{N}$ for forces, $\mathrm{pC}$ $\left(10^{-12} \mathrm{C}\right)$ for electric charges, and $\mathrm{V}$ for voltages. The torsional eigenfrequency of the mirror is $f_{0}=5370 \mathrm{~Hz}$. The moment of inertia $I$ is estimated from the given geometry and $K$ is accurately computed using standard tools of structural mechanics. On the contrary, the dissipation term is a delicate issue. Due to the presence of comb-finger actuation and of a large gap ( $350 \mu \mathrm{m}$ ) between the mirror plate and the substrate, the main contributions to dissipation are from shear flow in the comb fingers and the transport of mass induced by the large rotation of the mirror. Preliminary results have been obtained on a similar structure in [2] applying a state-of-the-art commercial code for the analysis of fluid-structure interaction and, independently, the formulation [22] implemented in Coventorware [23]; however, to increase the accuracy, in the present work, the $B$ value has been calibrated starting from the known maximum opening angle of the mirror in operative conditions. A single constant dissipation coefficient is utilized for all the analyses in this paper, yielding very satisfactory quantitative results (see, however, the comments to Fig. 14). An extensive numerical campaign is currently underway in order to improve the predictive capabilities.

\section{A. Capacitance Extraction}

A dedicated code based on integral equations has been applied to compute capacitance for one complete set of comb fingers, as represented in Fig. 2. The accuracy of the capacitance extraction phase is crucial for the success of the simulation procedure, and simple analytical formulas prove insufficient.

The capacitance is a function of the Euler angles of the rigid mirror. Fig. 3 plots the capacitance $C$ of this set of fingers in 


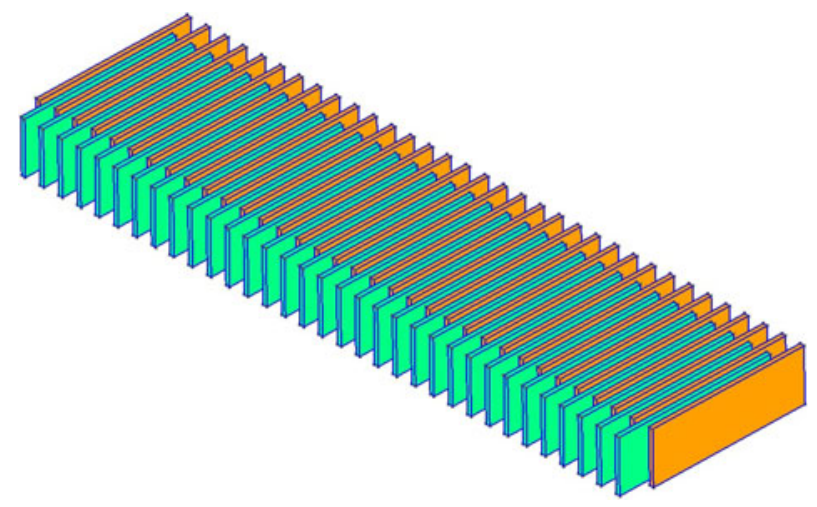

Fig. 2. Geometrical model of one complete series of interdigited fingers.

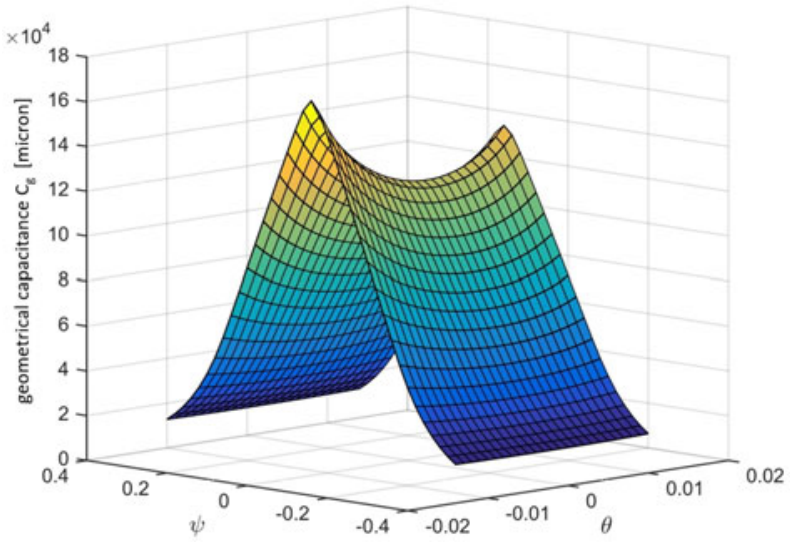

Fig. 3. Plot of the geometrical capacitance $C_{g}\left(C=\varepsilon_{0} C_{g}\right)$ for one of the four sets of interdigited fingers. $\psi$ and $\theta$ are two Euler angles of the mirror.

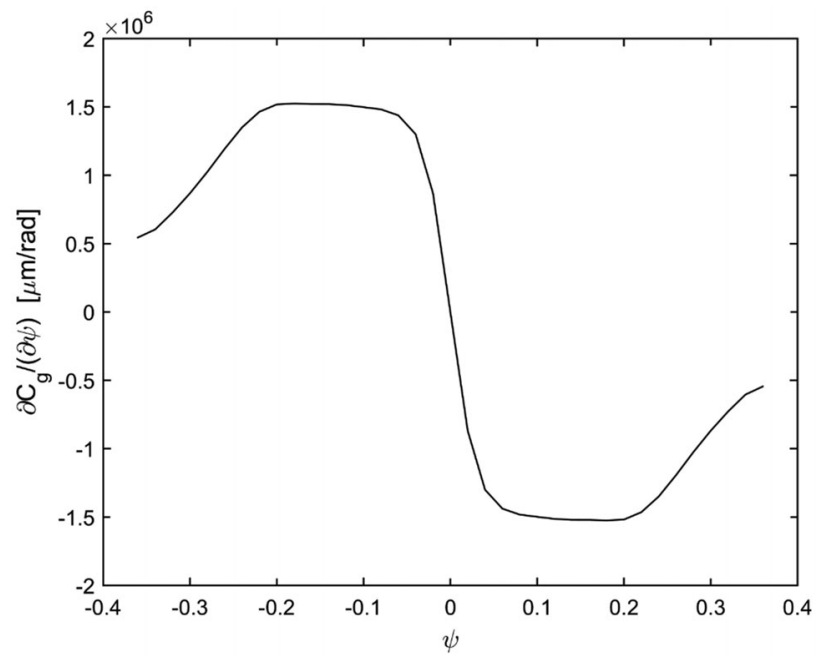

Fig. 4. Derivative of the total geometrical capacitance $C_{g}\left(C=\varepsilon_{0} C_{g}\right)$ with respect to the torsional angle, for $\theta=0$.

terms of $\psi$ and $\theta$, where $\theta$, not activated in this investigation, represents a rotation around the material axis orthogonal to the current configuration of the mirror surface. Starting from the data of Fig. 3, the derivative of the total capacitance with respect to $\psi$ is computed by means of numerical differentiation in the $\theta=0$ plane. The results are illustrated in Fig. 4 .

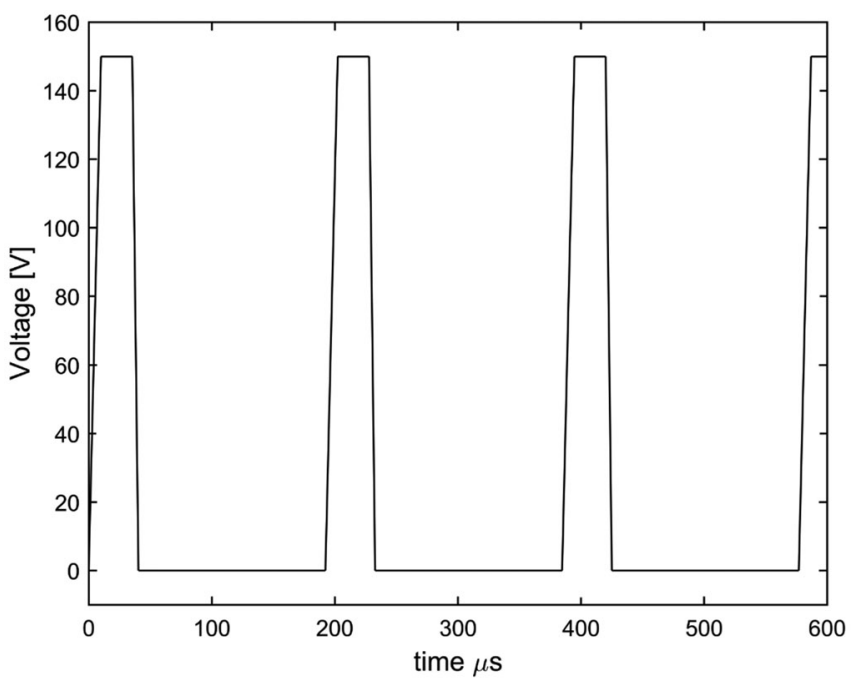

Fig. 5. Input voltage: square wave at $5300 \mathrm{~Hz}, 150 \mathrm{~V}$.

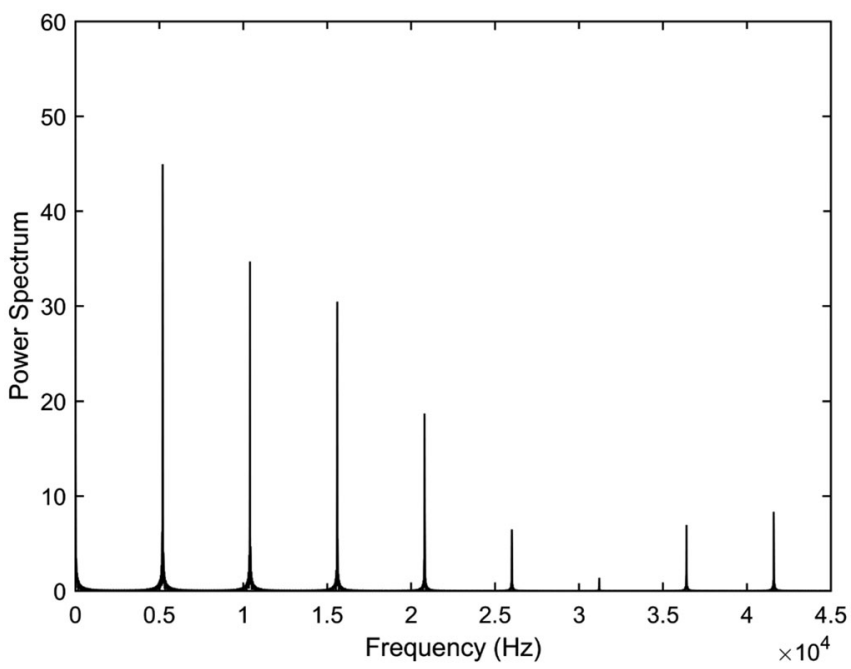

Fig. 6. Power spectrum for the square wave in Fig. 5.

\section{B. Mirror Actuation and Control}

In standard working conditions, the mirror is excited near the resonance peak, and the phase between the driving signal and the mirror is kept constant by a closed loop. The mirror is driven with a square wave at $150 \mathrm{~V}$ with a typical duration $<25 \%$ of the duty cycle. The square wave has finite ramp rates, which are typically set at 15 and $30 \mathrm{~V} / \mu$ s for the upward and downward ramps, respectively. Fig. 5 presents the square wave for a chosen frequency of $5300 \mathrm{~Hz}$, close to the natural torsional frequency of the mirror. It is worth stressing, for future discussion, that the FFT of the square wave contains almost all the multiples of the fundamental frequency, as evidenced in Fig. 6, illustrating the power spectrum.

\section{Analytical and Numerical Simulations}

The electrostatic forcing vanishes for $\psi=0$, and the plot of Fig. 4 can be roughly approximated with a linear term: $\left(\varepsilon_{0} / 2\right) \partial C / \partial \psi \simeq-K_{1}^{e} \psi, K_{1}^{e}>0$, for small $\psi$. Even if the mirror is excited with a square wave in actual operations, several experiments with a sinusoidal input are reported in Section IV. Hence, let us initially focus for simplicity on $V=V_{0} \cos \omega t$. 


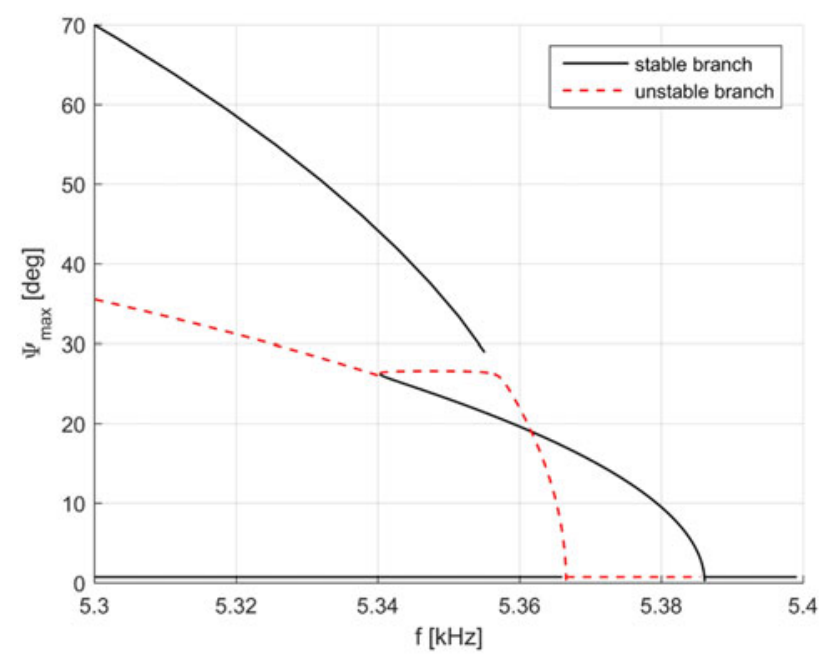

Fig. 7. Simulated stable and unstable branches for a cubic approximation of $\partial C / \partial \psi$ and $V=V_{0} \cos (\omega t), V_{0}=45 \mathrm{~V}$.

The forcing term in (1) becomes $-K_{1}^{e} V_{0}^{2} / 2(1+\cos 2 \omega t) \psi$ and

$$
I \ddot{\psi}+B \dot{\psi}+\left(K+K_{1}^{e} V_{0}^{2} / 2+K_{1}^{e} V_{0}^{2} / 2 \cos 2 \omega t\right) \psi=0 .
$$

In (2), $K_{1}^{e} V_{0}^{2} / 2$ is less than $0.8 \%$ of $K$ for $V_{0}=100 \mathrm{~V}$ and can be safely neglected in our applications. Equation (2) is a form of the well-known Mathieu equation [14], [16] with zero dissipation

$$
\frac{\mathrm{d}^{2} x}{\mathrm{~d} \tau^{2}}+(\delta+\varepsilon \cos 2 \tau) x=0
$$

where $\tau=\omega t$, parameter $\delta$ stands for $\omega_{0}^{2} / \omega^{2}$ (without considering the electrostatic stiffness; see comments above), and $\varepsilon$ is associated with the electrostatic forcing. Although the theoretical developments are quite involved, the linear Mathieu equation admits nontrivial solutions only in specific regions of the $\delta, \varepsilon$ space, called instability tongues. These tongues emanate from the $\varepsilon=0$ axis near drive frequencies corresponding to $\delta=n^{2}$, where $n$ is any positive integer. The region of practical interest is the tongue emanating from $\delta=1$. Even though theoretically there are infinitely many tongues, these almost never show up in real systems because they get narrow and are strongly reduced in the presence of dissipation (see, however, [16], where multiple tongues have been measured). For our purposes, this means that the physical system produces a nontrivial response only when the forcing frequency $2 \omega$ is equal to twice the eigenfrequency $\omega_{0}$ of the system.

However, the representation of the electrostatic forcing with a linear term is only a crude approximation of reality. A cubic term in $V^{2}$ can be added, leading to the generalized nonlinear Mathieu equation

$$
\frac{\mathrm{d}^{2} x}{\mathrm{~d} \tau^{2}}+(\delta+\varepsilon \cos 2 \tau) x-(\gamma+\gamma \cos 2 \tau) x^{3}=0
$$

which has been analyzed in [16]. The numerical simulation of (4) for the mirror parameters and a sinusoidal applied voltage is plotted in Fig. 7. It has been obtained applying the continuation approach described in the next section and reducing the dissipative term by two orders of magnitude for the purpose of comparison. It closely matches the results obtained in [16] for negative $\gamma_{3}=\lambda_{3}$ (in that paper notation). It is, however, clear that even a cubic approximation is realistic only for small angles, and it will indeed be shown, in Section V, that the real mirror response differs significantly. Also the approximation with a Gaussian function [18] does not prove sufficiently accurate for our purposes. Similar conclusions hold if we consider the square wave forcing of Figs. 5 and 6. The system admits nontrivial solutions whenever one of the Fourier components of $V^{2}$ is close to twice the natural frequency $\omega_{0}$ of the linear system, i.e., $10600 \mathrm{~Hz}$ in our case. Several pieces of evidences will be discussed in Section V.

\section{A. Numerical Continuation}

The numerical simulation of (1) can be performed both with the brute force approach and with a more refined continuation technique. In the former case, amplitude versus frequency plots are obtained by direct analysis in time. A sweep over the frequencies of interest is performed, and for each frequency, a sufficient number of cycles are simulated by direct integration to reach a steady state; the amplitude is then recorded, and the next frequency is addressed, using the final amplitude and phase of the previous analysis as initial conditions. This is a very robust technique, which, however, permits to simulate only the stable branches. On the contrary, the continuation approach [9], [24] with arc length control is more accurate and general and is preferred in this work, where a custom implementation of a simplified formulation has been utilized in order to address also the square wave excitation. The brute force simulation is only applied to get a reasonable initialization of the continuation procedure. The model in (1) is rewritten as a first-order nonautonomous differential system of equations in terms of the fictitious time $\tau=t / T$, with $T$ period of the forcing function

$$
\begin{aligned}
& y_{1}^{\prime}=T y_{2} \\
& y_{2}^{\prime}=-\frac{B T}{I} y_{2}-\frac{K T}{I} y_{1}-\frac{T \varepsilon_{0}}{2 I} \frac{\partial C}{\partial \psi} V^{2}(T \tau) .
\end{aligned}
$$

In (5), the prime denotes differentiation with respect to $\tau$, and $f=1 / T$ is the continuation parameter. Limiting our attention to periodic solutions with period $T$, we require $y_{1}(0)=y_{1}(1)$ and $y_{2}(0)=y_{2}(1)$. The segment [0-1] is partitioned into $N$ equal elements and the unknown functions $y_{1}, y_{2}$ are discretized over each element with quadratic Lagrangian shape functions. Equation (5) is solved with the method of orthogonal collocation, i.e., it is collocated at the two abscissae of the Gauss-Legendre orthogonal polynomial in each element. The unstable branches are followed using the Keller's pseudo-arclength continuation method [9], which imposes that, given a solution $y_{1}, y_{2}, f$, the increment $\Delta y_{1}, \Delta y_{2}, \Delta f$ is sought such that its projection along a specific direction (typically, the tangent to the $y_{1}, y_{2}, f$ manifold) equals a fixed arc length $\Delta s$.

\section{EXPERIMENTAL SETUP}

The monitoring of the opening angle of the electrostatic micromirror was obtained through the experimental measurement of the deflection of a laser beam incident on the device. The setup scheme for this test is reported in Fig. 8. In particular, the activation signal was produced by the function generator "Agilent 33521 A." He-Ne laser was preferred in order to have a spot size smaller than the mirror diameter without using other optical instrumentation for the beam collimation.

An attenuator was positioned in front of the laser to reduce the laser intensity and not saturate the camera. The mirror is mounted on an optical alignment bench in order to provide a $45^{\circ}$ laser beam incidence and reflection angle and a perpendicular projection of the reflected beam on the millimetric target. The resonant movement of the mirror spans a laser segment, which is acquired by a camera and processed through a ded- 

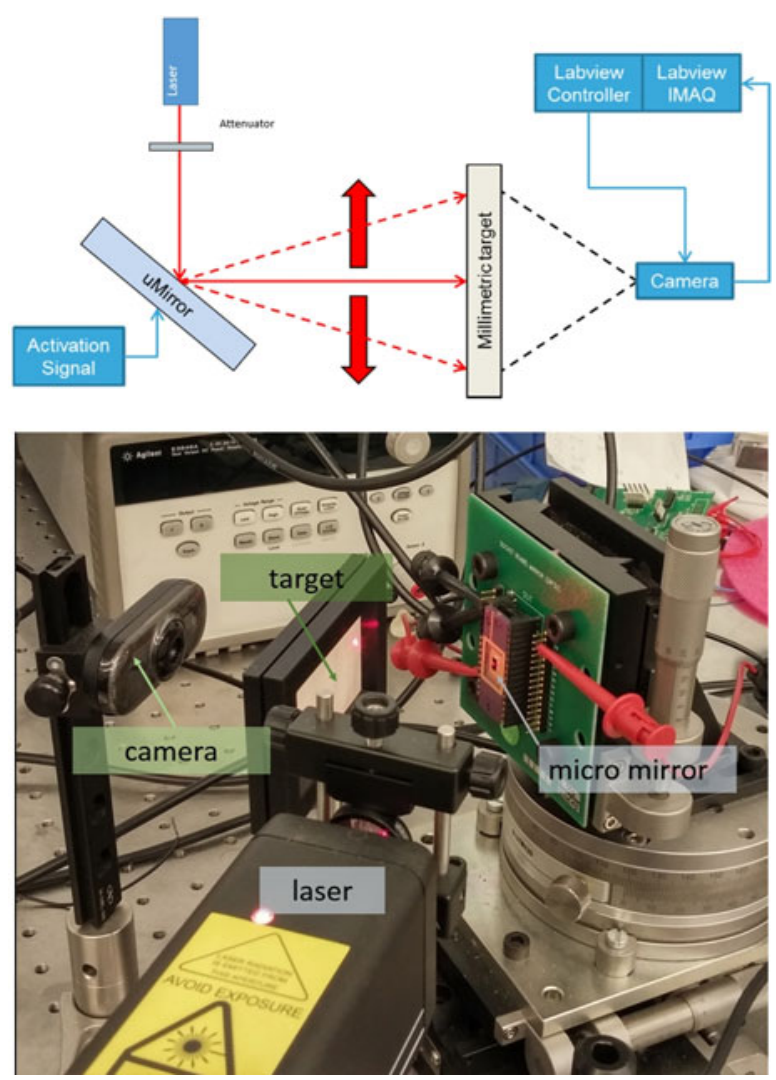

Fig. 8. Setup scheme (and photo) used for the dynamical characterization of the mirror photo of the setup.

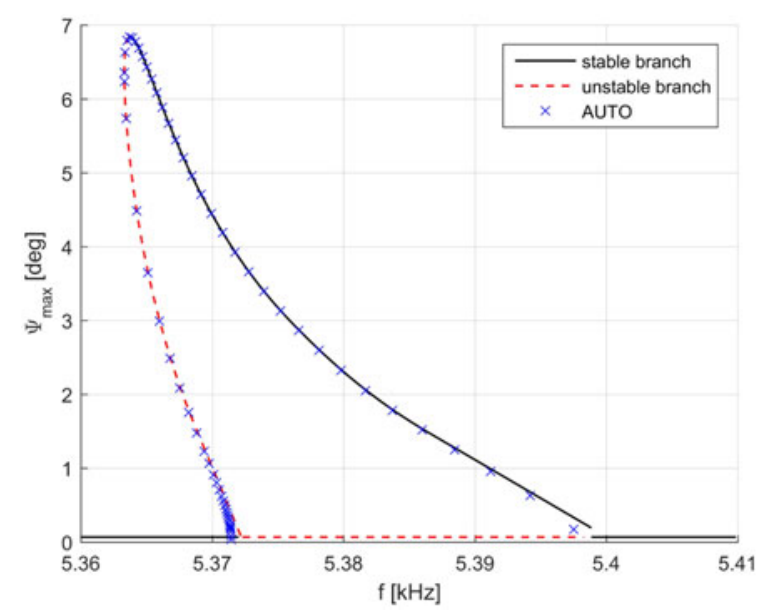

Fig. 9. Sinusoidal excitation at $5 \mathrm{kHz}, V_{0}=45 \mathrm{~V}$; comparison of the response computed with AUTO [24] (crosses) and with the present continuation approach.

icated Labview script. An accurate calibration of the camera was performed in order to obtain the relation between camera pixels and physical lengths. The mechanical opening angle is calculated through the following relation: $\psi_{\text {mech }}=1 / 2 \psi_{\text {opt }}=$ $1 / 2 \arctan (L / 2 d)$, where $L$ is the distance between the target and the mirror and $d$ is the laser segment length. The control software gives the possibility to set the scanning frequency, range, and step and direction of sweep (up or down).

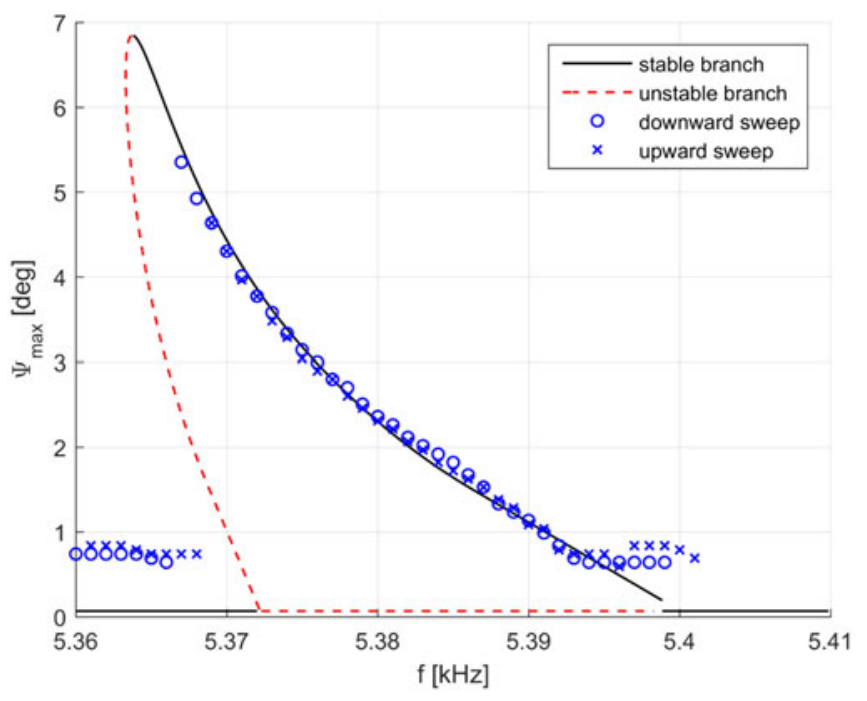

Fig. 10. Sinusoidal excitation at $5 \mathrm{kHz}, V_{0}=45 \mathrm{~V}$; experimental upward and downward sweep (discrete symbols) and numerical continuation (continuous line).

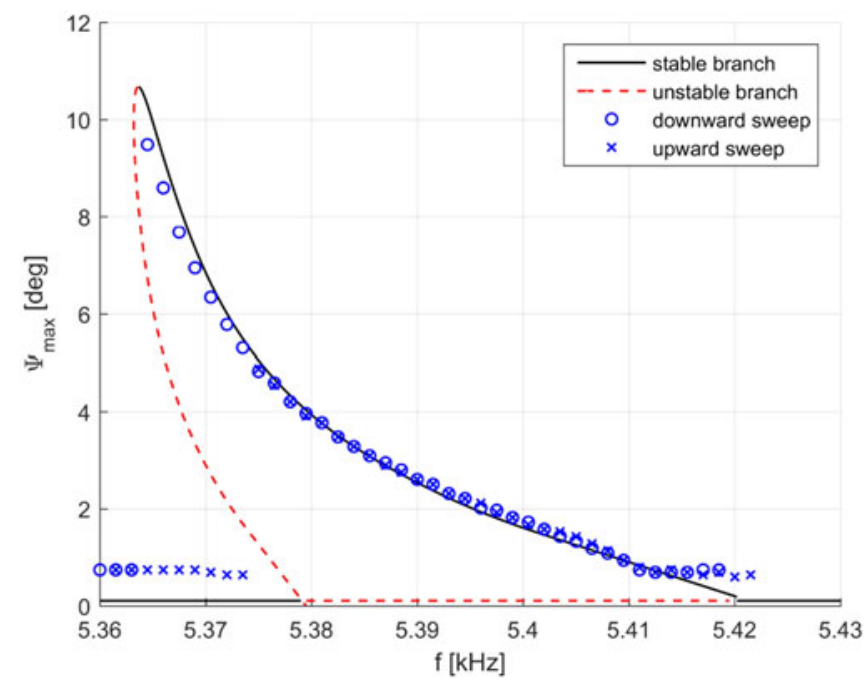

Fig. 11. Sinusoidal excitation at $5 \mathrm{kHz}, V_{0}=55 \mathrm{~V}$; experimental upward and downward sweep (discrete symbols) and numerical continuation (continuous line).

\section{COMPARISON OF NUMERICAL AND EXPERIMENTAL DATA}

\section{A. Sinusoidal Excitation}

In the case of pure tone excitation, the mirror has been actuated with sinusoidal voltages $V(t)=V_{0} \cos \omega t$ in two different ranges of frequencies: 2 and $5 \mathrm{kHz}$. The driving term $V^{2}$ contains the term $\cos 2 \omega t$ and the theory recalled in the previous section predicts that, in the absence of dissipation, a nontrivial response is obtained in regions of the space $\omega, V$ (instability tongues) emanating from the axis $V=0$ at $\omega=\omega_{0} / n, n>0$. In the $5-\mathrm{kHz}$ case, corresponding to $n=1$, the mirror shows the classical non trivial softening behavior: in this range, indeed, $2 \omega$ is close to $2 \omega_{0}$. Figs. $10-13$ collect all the experimental results in a broad range of peak voltages $V_{0}(45-90 \mathrm{~V})$. When available, both the upward and downward experimental sweep have been plotted, while the data are limited to the latter sweep in the other cases. Considering that only stable branches can be reproduced in the experiments, the agreement with numerical data is, in 


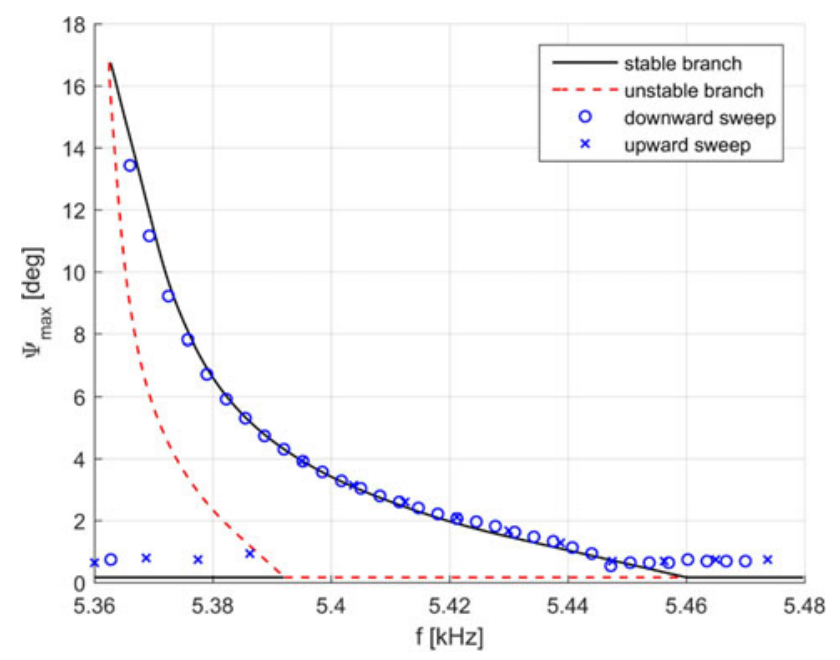

Fig. 12. Sinusoidal excitation at $5 \mathrm{kHz}, V_{0}=70 \mathrm{~V}$; experimental upward and downward sweep (discrete symbols) and numerical continuation (continuous line).

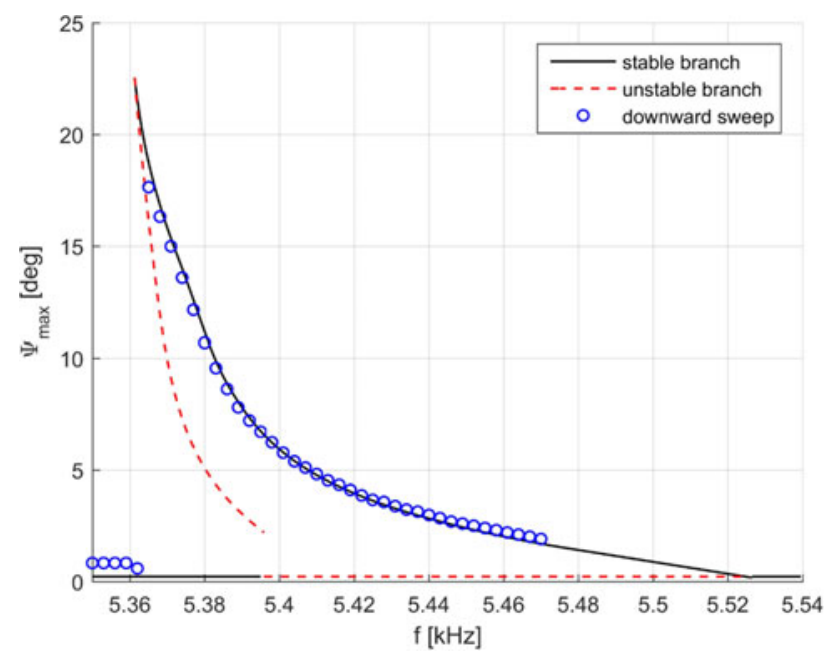

Fig. 13. Sinusoidal excitation at $5 \mathrm{kHz}, V_{0}=90 \mathrm{~V}$; experimental downward sweep (discrete symbols) and numerical continuation (continuous line).

general, impressive. For $V_{0}=45 \mathrm{~V}$ (see Fig. 9), the predicted response has been compared with AUTO [24], which represents the state of the art for continuation approaches. It is worth recalling that the model is almost exact, the only limitation being the simplified dissipative term $B \dot{\psi}$. From the comparison with experimental data, it is apparent that $B$ should be, in general, a (weak) function of $\psi$.

The comparison of the results presented above with the plot of Fig. 7, obtained with a cubic approximation for $\partial C / \partial \psi$, clearly indicates that an accurate representation of the electrostatic forcing for large $\psi$ is crucial. Tongues with $n>1$ are very narrow and difficult to measure experimentally and even simulate numerically. Moreover, in the presence of important damping as for the micromirror, tongues with $n>1$ exist only for large $V$. The experimental data for the maximum amplitude versus input frequency for the $2-\mathrm{kHz}$ case (i.e., $n=2$ ) and $V_{0}=90 \mathrm{~V}$ are displayed in Fig. 14. The measured response has a very limited amplitude and data are noisy. The shape of the curve, however, is as expected. In order to obtain a nontrivial result, the viscous coefficient has been lowered by one order of magnitude with

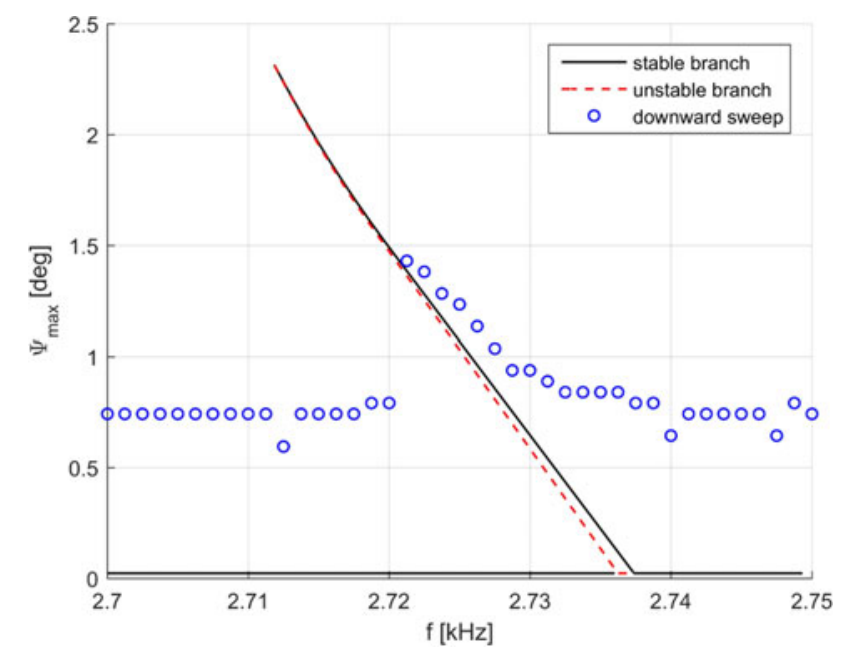

Fig. 14. Sinusoidal excitation at $2 \mathrm{kHz}, V_{0}=90 \mathrm{~V}$. Continuation approach (continuous line) and experimental data.

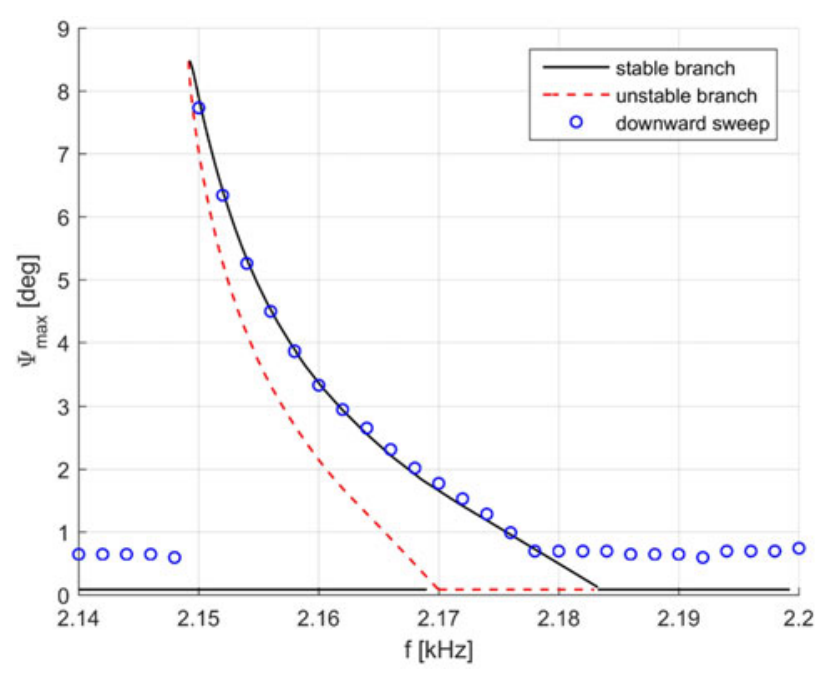

Fig. 15. Square wave excitation at $2 \mathrm{kHz}, V_{0}=110 \mathrm{~V}$; experimental downward sweep (discrete symbols) and numerical continuation (continuous line).

respect to the $B$ utilized for all the analyses of the paper, which seems coherent with the expectation that $B$ should be a function of the opening angle. However, the agreement between the frequencies and the slope of the curves is totally satisfactory. This represents one of the few experimental pieces of evidences available (see, however, [10]) of instability tongues with $n>1$.

\section{B. Square Wave Excitation}

When the square wave excitation is utilized, curious phenomena occur that could not be explained in the context of the classical harmonic resonance. When the input frequency is in the range of $2 \mathrm{kHz}$, no Fourier component for $V^{2}$ equals the torsional eigenfrequency, but the mirror is actually parametrically driven by the (fifth) Fourier component at $11 \mathrm{kHz}$, i.e., $2 \omega_{0}$, which is indeed rather small in amplitude. This explains why the maximum opening angles are limited in Figs. 15 and 16.

Moreover, the hysteresis loop is extremely narrow, which makes the continuation triacky. It is worth stressing that the continuation approach only works if the forcing and mirror response are periodic, which means that two periods of the square 


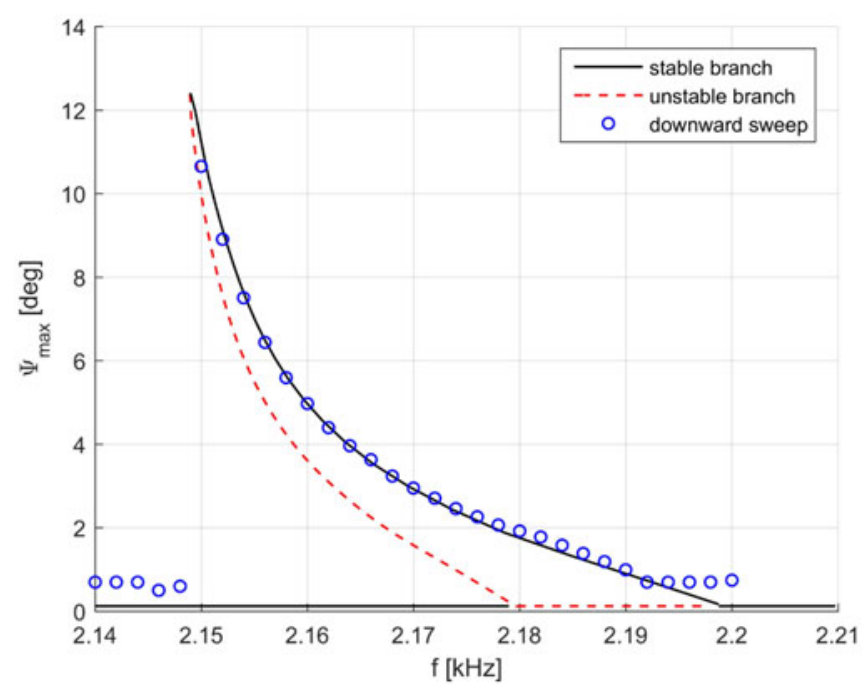

Fig. 16. Square wave excitation at $2 \mathrm{kHz}, V_{0}=130 \mathrm{~V}$; experimental downward sweep (discrete symbols) and numerical continuation (continuous line).

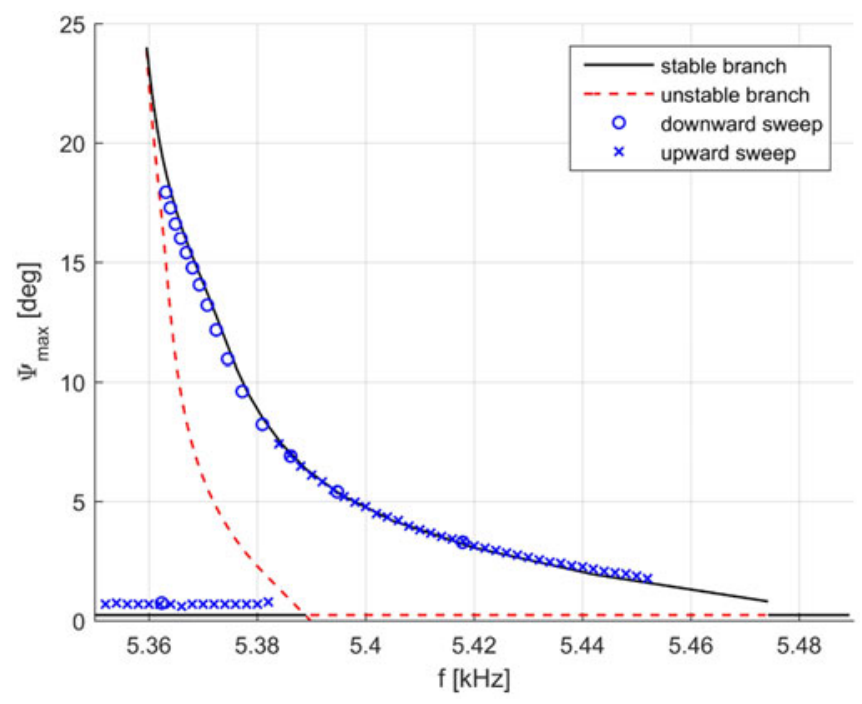

Fig. 17. Square wave excitation at $5 \mathrm{kHz}, V_{0}=110 \mathrm{~V}$; experimental upward and downward sweep (discrete symbols) and numerical continuation (continuous line).

wave and five periods of the mirror response must be simulated as one "global" period. Increasing the frequency of the input square wave, the torsional mode of the mirror is activated whenever there exists an integer $n$ such that $n \omega \simeq 2 \omega_{0}$. For instance, at $5 \mathrm{kHz}$, one has $n=2$, and at $10 \mathrm{kHz}, n=1$. These two cases have been investigated numerically and experimentally, the former being the normal operating mode of the mirror. Figs. 17 and 18 refer to the 5-kHz case. Finally, Figs. 19 and 20 present the comparison when the mirror is actuated with a square wave (with finite ramp up/down rates) in a frequency range around $10 \mathrm{kHz}$. In this case, in the FFT of $V^{2}$, the first high-energy Fourier component is at $2 \omega_{0}$ and, hence, energetically activates the mirror response. Even though the $V_{0}$ voltage utilized in this case is rather low, the mirror experiences very large rotations inducing partial failure of the experimental setup, which limits the validity of experimental data at the peak. It is worth stressing that the development of the ad-hoc code rapidly described in Section III-A has been stimulated specifically to

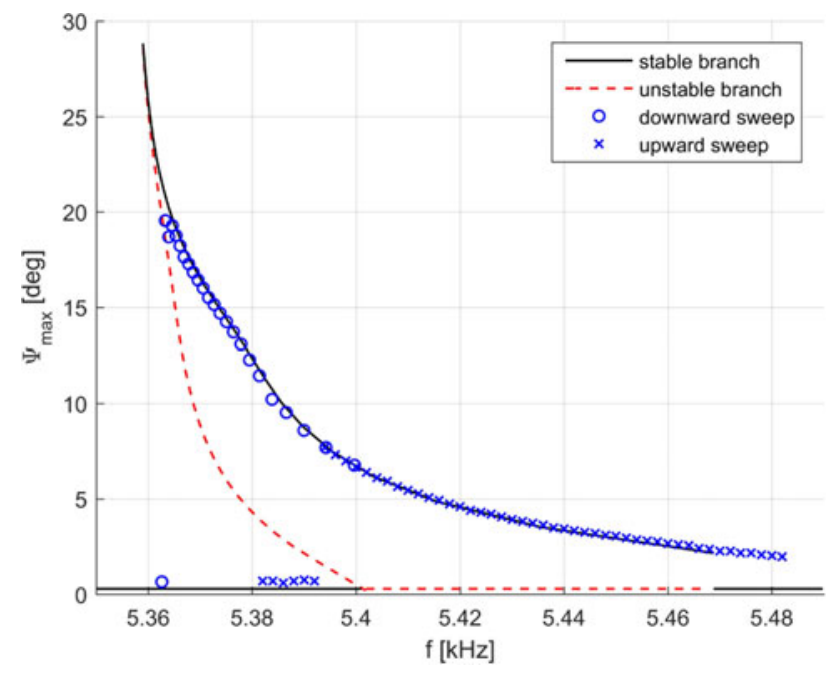

Fig. 18. Square wave excitation at $5 \mathrm{kHz}, V_{0}=130 \mathrm{~V}$; experimental upward and downward sweep (discrete symbols) and numerical continuation (continuous line).

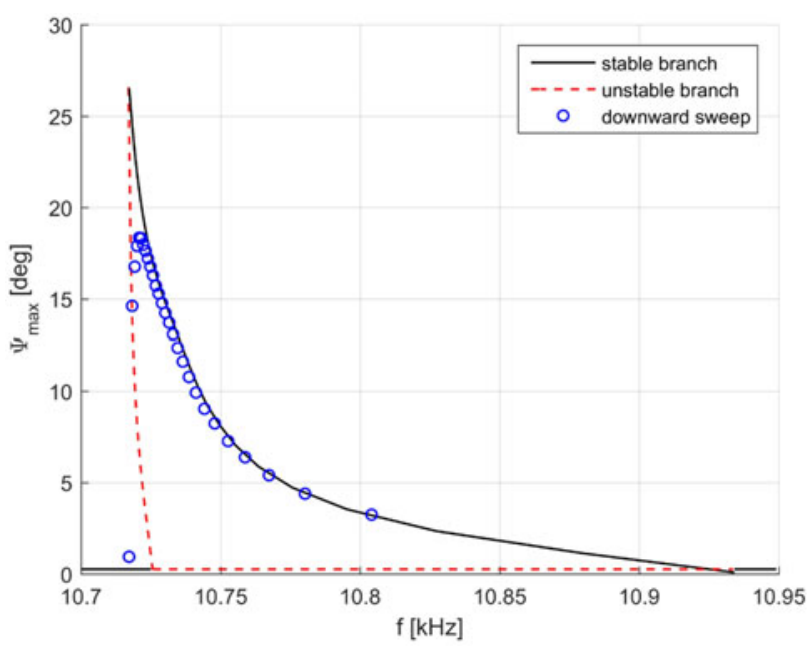

Fig. 19. Square wave excitation at $10 \mathrm{kHz}, V_{0}=90 \mathrm{~V}$; experimental downward sweep (discrete symbols) and numerical continuation (continuous line).

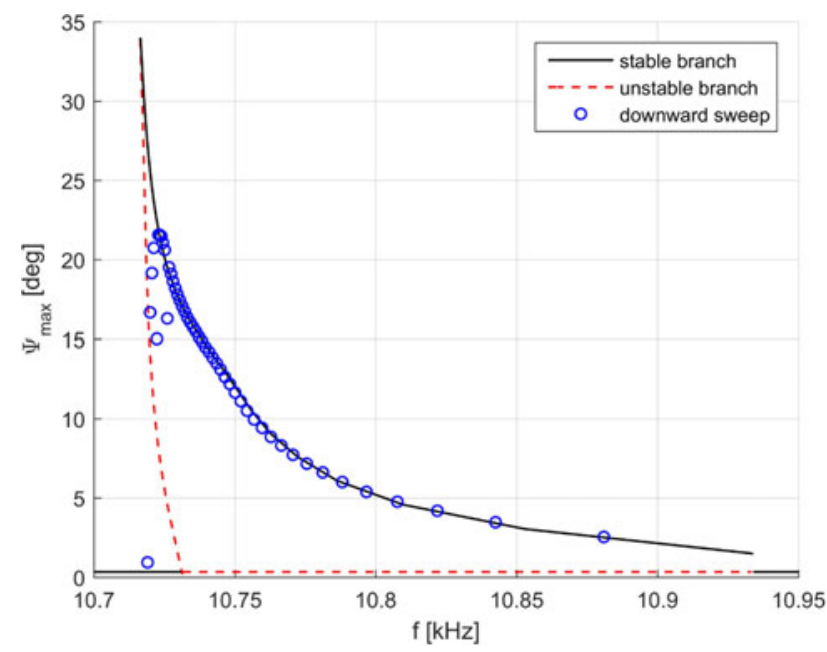

Fig. 20. Square wave excitation at $10 \mathrm{kHz}, V_{0}=110 \mathrm{~V}$; experimental downward sweep (discrete symbols) and numerical continuation (continuous line). 
avoid an approximate representation of the square wave as a finite sum of sinusoidal components inducing spurious Gibbs oscillations.

\section{CONCLUSION}

In this paper, we have thoroughly analyzed a typical electrostatically actuated torsional micromirror, and we have validated the numerical model with an extensive experimental campaign. The experimental data are reproduced with impressive accuracy. Parametric resonance is a well-understood phenomenon in nonlinear dynamics, and we have shown that the micromirror operates in this framework. In some cases, the experimental data appear as smoothed near the numerical peak. This might be due to the fact that in these cases, the instability tongue is typically very narrow and experimentally difficult to reproduce; also the dissipation coefficient injected in the numerical model is naive since a dependence on the opening angle is neglected. Investigations along these lines are ongoing. The nonlinear behavior of the main mode is also associated with fancy nonlinear coupling phenomena with spurious modes of the mirror, which are the topic of future works.

\section{REFERENCES}

[1] Lab4MEMS II, Micro-Optical MEMS, micro-mirrors and pico-projectors, ENIAC Project. [Online]. Available: http://www.lab4mems2.ite.waw.pl/

[2] R. Mirzazadeh, S. Mariani, and M. De Fazio, "Modeling of fluid damping in resonant micro-mirrors with out-of-plane comb-drive actuation," in Proc. Int. Electron. Conf. Sens. Appl., 2014, pp. 1-6.

[3] M. I. Younis, MEMS Linear and Nonlinear Statics and Dynamics. New York, NY, USA: Springer, 2011.

[4] X. M. Zhang, F. S. Chau, C. Quan, Y. L. Lam, and A. Q. Liu, "A study of the static characteristics of a torsional micromirror," Sens. Actuators A, Phys., vol. 90, pp. 73-81, May 2001.

[5] M. Taghizadeh and H. Mobki, "Bifurcation analysis of torsional micromirror actuated by electrostatic forces," Arch. Mech., vol. 66, pp. 95-111, Jan. 2014

[6] A. Caspani, C. Comi, A. Corigliano, G. Langfelder, V. Zega, and S. Zerbini, "Dynamic nonlinear behavior of torsional resonators in MEMS," J. Micromech. Microeng., vol. 24, Sep. 2014, Art. no. 095025.

[7] A. H. Nayfeh, Introduction to Perturbation Techniques. New York, NY, USA: Wiley, 1981

[8] W. Lacarbonara, Nonlinear Structural Mechanics. New York, NY, USA: Springer, 2013.

[9] D. Wagg and S. Neild, Nonlinear Vibration with Control. New York, NY, USA: Springer, 2015

[10] K. L. Turner, S. A. Miller, P. G. Hartwell, N. C. MacDonald, S. H. Strogatz, and S. G. Adams, "Five parametric resonances in a microelectromechanical system," Nature, vol. 396, pp. 149-152, Nov. 1998.

[11] D. Rugar, and P. Grutter, "Mechanical parametric amplification and thermomechanical noise squeezing," Phys. Rev. Lett., vol. 67, pp. 699-702, Jan. 1991

[12] M. Zalalutdinov et al., "Optically pumped parametric amplification for micromechanical oscillators," Appl. Phys. Lett., vol. 78, pp. 3142-3144, May 2001.

[13] D. W. Carr, S. Evoy, L. Sekaric, H. G. Craighead, and J. M. Parpia, "Parametric amplification in a torsional microresonator," Appl. Phys. Lett., vol. 77, Sep. 2000, Art. no. 1545.

[14] W. Zhang, R. Baskaran, and K. L. Turner, "Effect of cubic nonlinearity on auto-parametrically amplified resonant MEMS mass sensor," Sens. Actuators A, vol. 102, pp. 139-150, Dec. 2002

[15] J. F. Rhoads, S. Shaw, K. L. Turner, and R. Baskaran, "Tunable microelectromechanical filters that exploit parametric resonance," J. Sound Acoust., vol. 127, pp. 423-430, Oct. 2005

[16] J. F. Rhoads, S. W. Shaw, K. L. Turner, J. Moehlis, B. E. DeMartini, and W. Zhang, "Generalized parametric resonance in electrostatically actuated microelectromechanical oscillators," J. Sound Vib., vol. 296, pp. 797-829, Oct. 2006

[17] M. J. Thompson and D. A. Horsley, "Parametrically amplified z-axis lorentz force magnetometer," J. Microelectromech. Syst., vol. 20, no. 3, pp. 702-710, Jun. 2011.
[18] W. Shahid, Z. Qiu, X. Duan, H. Li, T. D. Wang, and K. R. Oldham, "Modeling and simulation of a parametrically resonant micromirror with duty-cycled excitation," J. Microelectromech. Syst., vol. 23, no. 6, pp. 1440-1453, Dec. 2014.

[19] B. E. DeMartini, J. F. Rhoads, K. L. Turner, S. W. Shaw, and J. Moehlis, "Linear and nonlinear tuning of parametrically excited MEMS oscillators," J. Microelectromech. Syst., vol. 16, no. 2, pp. 310-318, Apr. 2007.

[20] S. Krylov, I. Harari, and Y. Cohen, "Stabilization of electrostatically actuated microstructures using parametric excitation," J. Micromech. Microeng., vol. 15, pp. 1188-1204, Jun. 2005.

[21] T. Ono, H. Wakamatsu, and M. Esashi, "Parametrically amplified therma resonant sensor with pseudo-cooling effect," J. Micromech. Microeng., vol. 15, pp. 2282-2288, Dec. 2005.

[22] A. Frangi, G. Spinola, and B. Vigna, "On the evaluation of damping in MEMS in the slip-flow regime," Int. J. Numer. Methods Eng., vol. 68 , pp. 1031-1051, Dec. 2006.

[23] [Online]. Available: http://www.coventor.com/mems-solutions/products/ coventorware/

[24] E. J. Doedel and J. P. Kernévez, AUTO: Software for Continuation Problems in Ordinary Differential Equations with Applications. Pasadena, CA, USA: California Inst. Technol., 1986.

[25] M. I. Younis and A. H. Nayfeh, "A study of the nonlinear response of a resonant microbeam to an electric actuation," Nonlinear Dyn., vol. 31, pp. 91-117, Jan. 2003.

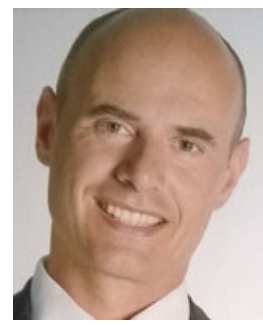

Attilio Frangi received the M.Sc. degree in aeronautical engineering and the Ph.D. degree in structural engineering from the Politecnico di Milano, Milan, Italy, in 1994 and 1998, respectively.

From 2001 to 2014, he was an Associate Professor in the Department of Mechanics, École Polytechnique, Palaiseau, France. He is currently a Full Professor with the DICA Department, Politecnico di Milano. He has authored more than 150 publications on topics of computational mechanics with focus on the simulation of multiphysics for microelectromechanical systems.

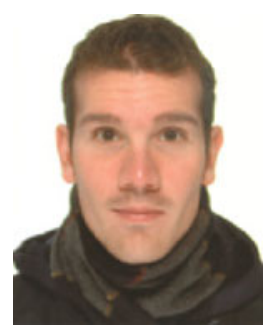

Andrea Guerrieri received the M.Sc. degree in structural civil engineering from the Politecnico di Milano, Milan, Italy, in 2015. His M.Sc. thesis studied the nonlinear dynamic response of suspension bridges subjected to aeroelastic effects. He is currently working toward the Ph.D. degree in the DICA Department, Politecnico di Milano, where he focuses on nonlinear dynamic phenomena of microsystems.

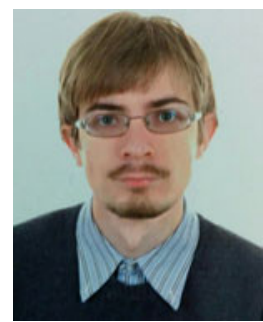

Roberto Carminati received the M.Sc. degree in physics from the University of Pavia, Pavia Italy, in 2011. During his M.Sc. studies, he worked on the optical characterization of nonlinear materials for telecommunications and holography.

$\mathrm{He}$ is currently a MEMS Design Engineer in the MEMS R\&D Group, STMicroelectronics, Cornaredo, Italy, where he is working on the development and industrialization of micromirror devices based on MEMS technology.

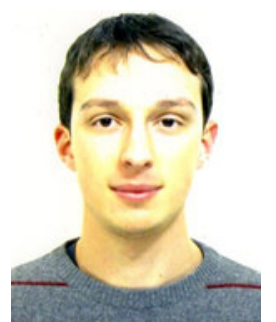

Gianluca Mendicino received the M.Sc. degree in physics from the Universit degli Studi di Milano, Milan, Italy, in 2015. During his M.Sc. studies, he worked on nuclear particle-phonon coupled states in the copper mass.

$\mathrm{He}$ is currently a MEMS Product Test Engineer in the MEMS Group, STMicroelectronics, Cornaredo, Italy, where he is working on the characterization team of the micromirror MEMS actuator. 